Meta

Journal des traducteurs

Translators' Journal

\title{
Ma boule ? C'en est assez pour devenir maboul !
}

\section{Philippe Desjardins}

Volume 37, numéro 2, juin 1992

URI : https://id.erudit.org/iderudit/002554ar

DOI : https://doi.org/10.7202/002554ar

Aller au sommaire du numéro

Éditeur(s)

Les Presses de l'Université de Montréal

ISSN

0026-0452 (imprimé)

1492-1421 (numérique)

Découvrir la revue

Citer cet article

Desjardins, P. (1992). Ma boule ? C'en est assez pour devenir maboul ! Meta, 37(2), 341-343. https://doi.org/10.7202/002554ar d'utilisation que vous pouvez consulter en ligne.

https://apropos.erudit.org/fr/usagers/politique-dutilisation/ 


\section{MA BOULE ? C'EN EST ASSEZ POUR DEVENIR MABOUL!}

Face à ma boule d'ivoire - que n'est-elle de cristal - la question se pose, forcément, de savoir comment s'appelle ce machin: une boule sculptée, remplie de plusieurs autres boules de taille décroissante. Cette boule remontant bien loin dans le temps (XIve siècle), il y sûrement une expression usitée, en français comme en anglais, pour la désigner, mais laquelle?

Aux Renseignements terminologiques de la fonction publique, Marc Laforge, orfèvre en la matière, puisqu'il en sculpte en bois, opterait, faute de mieux malgré ses recherches, pour boules «encagées / intercalées». La boule étant une figure géométrique, et deux têtes valant mieux qu'une, pourquoi pas un coup de fil à un ingénieur ami ? Fidèle à sa profession, il avance «boules concentriques», mais concède que «sphères» irait bien aussi. Des fois que ça pourrait aider, voyons, du côté anglais, la confusion qui semble régner sur cette boule-sphynx.

À la page 163 de The Modern Book of Whittling and Woodcarving, E. J. Tangerman, McGraw-Hill, 1973, rubrique «Carved Ivories», le texte accompagnant l'illustration de la boule en question dit : «Nested spheres - 17, one inside the other».

L'Oxford Dictionary, deuxième édition, 1989, définit nested ainsi : «Such that each item or constituent contains or is contained within another similar item in a hierarchical arrangement», alors que l'ouvrage 12,000 Words - A Supplement to Webster's Third New International Dictionary, 1986, en donne la définition suivante: «Forming a sequence or hierarchy with each member contained in or containing the next.» Rien de plus clair. Dans le Harrap's Standard French and English Dictionary, on trouve les expressions nested boxes et nested cones rendues par «caisses emboîtées» et «cônes 
emboîtés» respectivement; avec Le Robert \& Collins (qui ne donne pas nested), il rend nest of tables par «table gigogne».

À la page 834 de la Chamber's Encyclopedia, 1973, on trouve concentrically carved balls.

L'Encyclopedia Britannica, 1973, donne, page 814, 2e colonne : balls-within-balls, expression répétée, mais sans traits d'union, sous l'illustration de la planche V. Il y est aussi question de Canton work, expression que Tardy a rendue par «travail cantonais», dans Les Ivoires (voir page 3).

Les expressions se diversifient en raison directe du nombre des ouvrages consultés. L'auteur britannique Charles Maskell, dans son livre intitulé Ivories, Editions Charles E. Tuttle, Tokyo, 1986, au chapitre «Chinese Ivories», emploie l'expression puzzle-ball dans la légende de l'illustration, mais sans trait d'union dans le texte.

The Collector's Encyclopedia, Collins, 1974, ouvre de nouveaux horizons : «devil's workball or puzzle ball. Concentric, free-moving spheres, with delicate pierced-work design, carved from small, solid ball of ivory. Made in China, particularly Canton, from 14th century». Bizarre qu'il définisse ball par sphere. L'ouvrage Warman's Antiques \& Their Prices utilise aussi le mot puzzle.

La concision ne paraissant pas caractériser le milieu, voici non pas l'appellation mais plutôt la description relevée dans The Complete Antiques Price List, Ralph and Terry Kovel, 6e édition, 1973, page 284: «Ivory Ball, Patience, Eight Movable Balls Within an Outer Ball.» Que signifie ici le mot patience? Dans le contexte, sa présence n'explique rien. Malgré les recherches ailleurs, son sens reste hermétique: tous les dictionnaires et encyclopédies consultés à ce sujet, chiches d'éclaircissements, sont muets comme des carpes. On se heurte à un mur jusqu'à ce que le hasard, sous forme d'une vente aux enchères, ouvre la voie à la lumière grâce à un trimestriel aujourd'hui disparu. The Antique Trader, Summer 1973, page 86, donne la description suivante: «Patience ball with seven movable balls within, on carved stem.» Une description analogue avait déjà paru page 84 de la livraison Spring 1973. C'est plus clair et concret que dans Kovel, ça explique tout. D'autre part, page 85 de la livraison Winter 1972 du même périodique et désignant la même sphère, on lisait : «Puzzle ball, $3^{1 / 2} 2^{\prime \prime}$ diameter, on carved base.»

Pour conclure, et peut-être empirer la confusion qui règne du côté de chez John Bull (encore boule !) - et de l'Oncle Sam aussi ? - l'ingénieur ami a relevé «an ivory concentric balls novelty, xIXth century...» page 527 du Miller's International Antiques Price Guide, Judith and Martin Miller, Vicking Penguin Inc., 1987. Voilà donc trois substantifs: spheres, balls, workballs, et sept qualificatifs: nested, carved, movable, : concentric, puzzle, devil's, et à la rigueur patience, mis à contribution pour nommer la boule.

Regardons maintenant du côté français, où la confusion ne paraît pas moins étendue. Au départ, il faut écarter "gigogne» sans merci, toutes les choses dites «gigogne» (poupées, tables, etc.) sortent les unes des autres, tandis qu'en l'occurrence les boules sont irrémédiablement captives.

«Concentrique» convient sans aucun doute, mais ne semble pas suffire sans s'accompagner de précisions.

Le Webster's New Collegiate Dictionary, 1951, définit concentric ainsi : «Having a common center, as circles or spheres one within the other», les Grand Robert et Larousse étant loin d'être aussi clairs et concrets sur le mot «concentrique».

Philippe Delaune, dans Les Ivoires, Éditions Jacques Grancher, 1987, dit page 34 à propos des divers modèles de boules figurant page 33 (donc aucune méprise possible): «trois pièces à pans percés de fenêtres rondes avec globes mobiles à l'intérieur». C'est ben beau ça, mais encore une fois, c'est une description, non une désignation. Plus loin, 
page 83, Delaune écrit : «Pour les ivoires japonais et chinois des XVII' et XIX ${ }^{\mathrm{e}}$ siècles, il faut compter [...] des globes mobiles encastrés à partir de 230 F.» Retenons les mots «globes», «mobiles» et «encastrés».

L'Encyclopedia Universalis, 1989, au mot ivoire, rubrique «Chine», donne les précisions suivantes : «c'est pourquoi certaines pièces ont pris le nom du port de Canton, telles ces balles concentriques, appelées aussi balles chinoises. Leurs modèles semblent avoir été les boules s'imbriquant les unes dans les autres fabriquées par les Européens». Ici encore, aucune équivoque sur la nature de l'objet visé.

À la page 159 du volume premier de l'ouvrage Les Ivoires, de Tardy, 1977, il est écrit: «Les boules sont faites au burin à ciseau centré. On détache d'abord la boule la plus petite, puis la suivante. Les Chinois arrivaient à faire tenir 20 boules l'une dans l'autre. XVII ${ }^{\mathrm{e}}-\mathrm{XVII} \mathrm{e}^{\mathrm{e}}$ s.» À la page $284 \mathrm{du}$ volume 2 , Tardy écrit : «série de boules évidées [...] contient environ 18 sphères intérieures». À la page suivante, 285 , il ajoute : «boule sculptée de personnages et paysages [...] Elle contient 13 autres boules au moins». Aux pages 280-281, on avait déjà relevé, à propos des mêmes boules ou sphères, l'expression «travail cantonais», qui rejoint ce qu'on vient de lire dans Universalis, à savoir que «certaines pièces ont pris le nom du port de Canton».

Le Dictionnaire des Antiquités et de la Brocante, Larousse, 1983, emploie, page 283, la longue expression «boules ajourées enfermées les unes dans les autres».

On vient de voir que, dans Tardy, ce sont les mots «boules» et «sphères» qui se côtoient, tandis que Delaune parle de «globes» et, dans Universalis, ce sont les mots «balles» et «boules» qui alternent.

Les Muses, Grange Batelière, Paris, tome 8, page 2692, parle des «fameuses balles chinoises concentriques».

Nous voici donc en présence des mots: balle, boule, globe, sphère pour les substantifs, et concentrique, mobile, évidé, encastré, sculpté, ajouré, enfermé, cantonais, chinois, de Canton pour les qualificatifs. Ouf!

Soit dit en passant, il semble que ces balles, boules, globes, sphères ne sont jamais sculptées en tant qu'objets distincts, mais plutôt comme parties intégrantes d'ensembles qui, en ayant la forme, porteraient le nom de «tour». Comme ces sphères secrètes assurent la retraite la plus sûre à quelque homuncule qui désirerait s'isoler, à l'abri de toute intrusion, peut-on conclure que Sainte-Beuve s'en serait inspiré pour créer ou répandre l'expression «tour d'ivoire»?

À l'instar de Galilée s'écriant : «Et pourtant, elle se meut !», on est en droit de se dire: «Et pourtant...» il doit bien y avoir, pour désigner la fameuse boule, un mot privilégié, consacré par l'usage, comme c'est le cas, par exemple, pour le bilboquet ou poussah, ou encore le palanquin ou houdah.

Ma recherche épuisée et ayant débordé ma sphère sans aboutir à une conclusion nette, j'ai renvoyé la balle dans le camp du Service des renseignements terminologiques pour qu'il jette un peu de lumière sur ce globe, de crainte que je n'en perde la boule! Je la relance maintenant, la balle, aux lecteurs de Meta. En place pour le quadrille! Et que ça saute... la balle ! Calling all Devil's ball fans!

PHILIPPE DesJaRdins 